\title{
Leak Localization in Water Distribution Networks using Deep Learning
}

\author{
Mohammadreza Javadiha ${ }^{1}$, Joaquim Blesa ${ }^{1,2,3}$, Adrià Soldevila ${ }^{1}$ and Vicenç Puig ${ }^{1,2}$
}

\begin{abstract}
This paper explores the use of deep learning for leak localization in Water Distribution Networks (WDNs) using pressure measurements. By using a training data set including enough samples of all possible leak localizations, a Convolutional Neural Network(CNN) can be used to learn the different pressure maps that carachterized each leak localization. The generalization accuracy has validated and evaluated by means of a testing data set. All of considered training, validation, and also testing data include leak size uncertainty, nodal water demand uncertainty and sensor noise. An innovative approach is proposed to convert every pressure residuals map to an image in order to apply a CNN. In addition with the purpose of filtering the effects of uncertainty and noise a time horizon Bayesian reasoning approach is used over each time instant classification output by the CNN. The Hanoi District Metered Area (DMA) is considered as a case study to illustrate the performance of the proposed leak localization method.
\end{abstract}

Keywords: Water distribution networks, leak localization, Deep Learning, fault diagnosis, Bayesian technique.

\section{INTRODUCTION}

Water leaks are present to some extent in all Water Distribution Networks (WDNs) and are estimated to account up to $30 \%$ of the total amount of extracted water [1]. This is a very significant amount since water is a precious resource in many parts of world that try to satisfy water demands of a growing population and sometimes in drought periods which are increased by the climate change.

Leak detection and localization in WDNs is a very active area of research, see [2] for a recent and extensive review.

Some of the recent proposed leak localization methods use pressure sensors inside the WDN and the inlet flow sensor of the WDN. Usually, WDNs are divided into different sectors also known as District Metered Areas (DMAs). The water companies consider as a feasible approach the possibility of installing only a few pressure sensors inside the DMAs, which are cheaper and easier to install and maintain than flow sensors. On the other hand, flow sensors at DMAs inlets are usually installed for control and billing purposes. In [3], a model-based method that relies on the pressure measurements and leak sensitivity analysis was proposed. In this methodology, pressure residuals, i.e. differences between pressure measurements provided by sensors and the corresponding estimations obtained by using the hydraulic network model, are used. These residuals are computed online and compared against associated thresholds that take into

\footnotetext{
${ }^{1}$ Supervision, Safety and Automatic Control Research Center (CS2AC) of the Universitat Politcnica de Catalunya, Campus de Terrassa, Gaia Building, Rambla Sant Nebridi, 22, 08222 Terrassa, Barcelona.

${ }^{2}$ Institut de Robòtica i Informàtica Industrial (CSIC-UPC). Carrer Llorens Artigas, 4-6, 08028 Barcelona.

${ }^{3}$ Serra Húnter Fellow
}

account the effects of modeling uncertainty and noise. When some of the residuals exceed their thresholds, the residuals are matched against the leak sensitivity matrix in order to discover which of the possible leaks is present. Although this approach has good efficiency under ideal conditions, its performance decreases due to the nodal demand uncertainty and noise in the measurements [4], [5].

In the last years artificial intelligence methods have been applied for leak localization purposes as in [6] where it was proposed a method to localize leaks using Support Vector Machines (SVM) that analyzes data obtained by a set of pressure sensors of a pipeline network to localize and estimate the size of the leak. In a similar way, more recently, the use of $k$-Nearest Neighbors, neuro-fuzzy and Bayesian classifiers for leak localization has been also proposed in [7], [8] and [9] respectively. The performance in leak localization of some of these methods has been assessed in [10].

On the other hand, nowadays there is an emerging research about the use of Deep Learning (DL) methods in fault diagnosis of complex systems (see [11] for a recent overview). This work is a first attempt to deal with the leak localization problem (particular case of fault diagnosis) in WDNs using DL methods.

The paper is organized as follows. Section II presents in detail the proposed method. Section III details the application of the method to a simplified WDN of the real WDN of Hanoi (Vietnam). Finally, the main conclusions of the work are presented in Section IV.

\section{Methodology}

This paper proposes a leak localization method in WDNs that tries to exploit Deep Learning potentials for analysis and exploration through the map representation of pressure residuals of the WDN. The leak detection is out of the scope of this work since it will be assumed that the leak detection is performed by an efficient method (as the night flow analysis [1]). So the proposed leak localization method will be triggered when a leak has been detected in the WDN.

\section{A. Architecture}

The proposed method follows the general scheme for online model-based Fault Detection and Isolation (FDI) strategies, where residuals are generated as the difference of actual sensor measurements (in this case pressure measurements in $n_{p}$ inner nodes of the network $S_{1}, \ldots, S_{n_{p}}$ ) and the estimation of these values provided by a model of the WDN that considers no-leak conditions. When a leak is detected in the network, the pressure residuals are evaluated to determine 
what is the most probable node in the WDN where the leak has been occurred.

The particular scheme of the proposed method is depicted in Fig. 1. As it can be seen in this figure, pressure estimations in inner nodes are computed by an hydraulic simulator of the network. The hydraulic simulator should include the information of all the components of the WDN: pipes (graph, distances, coefficients), nodes ( $\mathrm{x}, \mathrm{y}$ and $\mathrm{z}$ positions), valves, etc. In addition, estimated node demands $\hat{d}_{1}, \ldots, \hat{d}_{n_{n}}$, where $n_{n}$ is the total number of inner nodes, from the measured total inlet flow $F_{\text {in }}$ and consumers historical billing information are provided to the hydraulic simulator as well as other measured boundary conditions.

Once $n_{p}$ residuals $r_{1}, \ldots, r_{n_{p}}$ associated to the pressure sensors installed in $n_{p}$ inner nodes, an estimation of the all possible $n_{n}$ residuals associated to all the nodes of the network $\hat{r}_{1}, \ldots, \hat{r}_{n_{n}}$ is computed using an interpolation method. This task is carried out by means of the Kriging method that is a well-known interpolation approach in the area of geostatistics [12] and that has recently applied to WDNs with successful results [13]. This interpolation method uses the topological and hydraulic information between all the nodes of the WDN to compute the estimation of the residuals in all the nodes of the network $\left(n_{n}\right)$ with the $n_{p}$ generated residuals associated to the $n_{p}$ pressure residuals installed in the network. The on-line residual estimation in all inner nodes of the network and the topological coordinates of all the nodes are used to generate a residual map of the network. Converting these map to 2-D pictures allows to create an image data associate to each leak location. Then, using a Deep Learning Convolutional Neural Network (CNN) for image classification allows us to determine what is the most probable node where the leak is present.

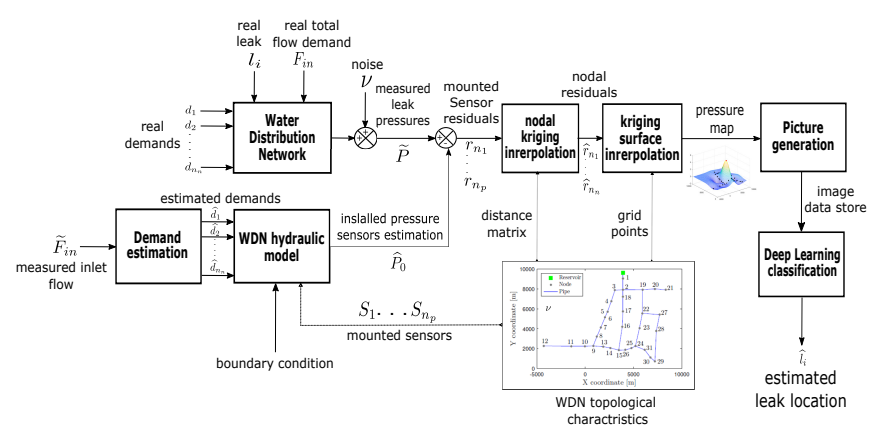

Fig. 1: Leak localization scheme using Deep Learning.

\section{B. Data generation}

The main problem of model-based FDI methods is that residuals are not only affected by faults but also by modelling errors. So, the accuracy of fault diagnosis using these methods depends on the quality of the model. In the proposed method, the main source of error in model-based pressure residuals is the difference of actual nodal demands $\left(d_{1}, \ldots, d_{n_{n}}\right)$ and its estimations provided to the hydraulic simulator due to demand uncertainty in predictions. Another source of error in pressure residuals is additive noise in sensor measurements. Moreover, the interpolation errors of the Kriging method also affect the accuracy in the leak localization task.

Machine learning techniques, and in particular CNNs need to use some off-line data for training a model to predict further data. The data store set is split into training, validation and testing subsets. The training subset is used for adjusting the weights and biases that compound the different layers of the CNN. Simultaneously selecting some random data as a validation subset avoids over-fitting during the training process, and the purpose of testing subset is the evaluation of prediction accuracy. The proposed method based on DL CNN should be able to localize any leak at any node despite of all possible sources of errors (such as demand estimation uncertainty, sensor noises and interpolation errors). The offline data subsets should include all possible leaks but also all possible errors. As in WDNs is not feasible to obtain real data for all the possible leak locations and it is not possible to manipulate the model (set of non-linear implicit equations) to have an analytical expression that allows to consider the effect of leaks and errors in pressure residuals, off-line synthetic data is generated as proposed in [9]. The scheme of the data generation method deployed in this paper is presented in Fig. 2 that is similar to the fault diagnosis scheme of Fig. 1 but substituting the real WDN by its hydraulic simulator. In this way, all the possible leak scenarios can be simulated considering demand uncertainties and other sources of errors in the generated images.

The images obtained in the off-line data generation method can be split into $n_{n}$ folders with respect to the number of associated inner node where the leak has been generated. In this way, every folder has a set of images that correspond to residual surfaces related to various operating conditions for the same leak location. Each pixel of the pictures represents a point of the network and its color is related to the residual magnitude. It is important to make all pictures in a same range by performing a global normalization as a prepossessing stage. Among all the folders, and all the pictures inside, the maximum and minimum values of the pixels of the raw pictures, denoted as $a$ and $b$ respectively, have been computed. This action followed by a multiplication bound to set the range of values between 0 and 255, outcomes achieving a standard format of picture desired for the image classification processing. In black and white, image data stores values between 0 and 255, where the extreme values represent pure white and black, respectively. Mathematically, the normalization of a generic raw pixel $x_{i, j}^{p}$ to $\bar{x}_{i, j}^{p}$ (where $p$ denotes the picture number and $i$ and $j$ the row and column position of the pixel in the picture) is computed by means

$$
\bar{x}_{i, j}^{p}=255 \frac{x_{i, j}^{p}-a}{b-a}
$$

where $a$ is computed as

$$
a=\max _{\substack{i \in\left\{1, \ldots, N_{I}\right\} \\ j \in\left\{1, \ldots, N_{J}\right\} \\ p \in\left\{1, \ldots, N_{P}\right\}}} x_{i j}^{p}
$$


with $N_{I}$ and $N_{J}$ the number of rows and columns of the pictures respectively, and $N_{P}$ the number of pictures. $b$ is computed as in (2) but replacing "max" by "min".

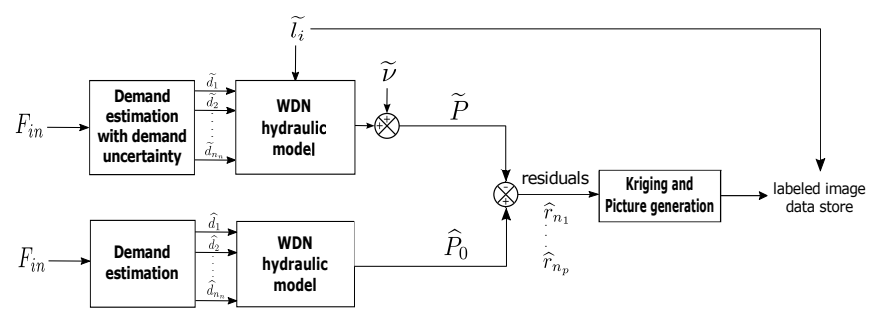

Fig. 2: Data generation scheme.

Accordingly, the off-line picture generation is proposed for the purpose of providing a comprehensive data set containing leaks in all the nodes of the network and considering different leak magnitudes, operating conditions, demand uncertainties, measurement noises in pressure sensors and interpolation errors. The obtained picture set should be suitable to train, validate and test the DL CNN image classifier.

\section{Deep Learning leak localization}

The task of the DL classifier in the leak localization scheme presented in Fig. 1 is given a picture that represents the on-line residual distribution in the network determine what is the most probable leak location. DL CNNs [14] is an emerging form of machine learning outstanding in image processing and voice recognition tasks. A deep learner is expert to find the unique characteristics of the images belonging to the same labeled class using a pre-designed structures composed by layers with distinct function. The objective is to attain the corresponding weights and biases in order to calibrate the relevant deep model to make relationship between batches of mixed input images to their respective label (leak location). This type of machine learning classifier needs a lot of data to archive the desired performance.

Given a picture at instant time $k$ denoted as $x^{k}$ that represents the residual distribution in the network at this time instant, the CNN will provide the probabilities that this picture has been produced at each inner node

$$
P\left(x^{k} \mid l_{i}\right) \quad i=1, \ldots n_{n}
$$

where $i$ denotes the leak location candidate. Then, the most probable leak location $\hat{i}$ can be computed as

$$
\hat{j}=\max _{i \in\left\{1, \ldots, n_{n}\right\}} P\left(x^{k} \mid l_{i}\right)
$$

To achieve a precise classification, an appropriate framework model should be developed with suitable adjustment settings.

1) Framework architecture: In order to design an appropriate CNN architecture (Fig. 3), several sorts of layers should be set consecutively either in serial or parallel for more complex missions. Selection of configuration, numbers, type and internal setting options are highly dependent on size, complexity, and volume of labels and data [15]

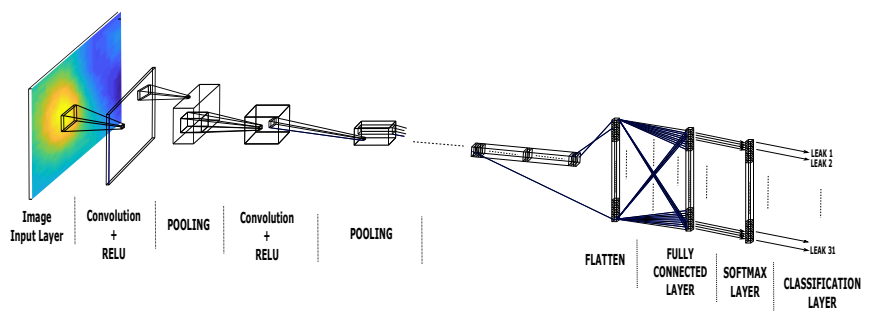

Fig. 3: Deep Learning Convolutional Neural Network architecture.

- Image input layer specifies the image pixel sizes construction derived by width, height, and the channel size 1 for grayscales, 3 for color images(RGB).

- Convolution2dlayer generally placed after Input layer. This layer consists of several square filters with the same size to detect features of pictures such as edge, spots, blur. These filters normally are specified with the same depth as input images. Each filter or kernel act such a neuron in a layer. By slipping the filters longitudinally and transversely over the input image by pixel jump step named "stride" and performing convolution by moving step, layer output could retained. In order to perfectly fitting, a zero padding strip is usually added to the input image.

- Batch normalization layer is used between convolutional layers and every term containing non-linearities. This layer increases training speed and decreases initialization sensitivity.

- Rectified Linear Unit (ReLU) layer retains the positive values, and converts the negative values to zero.

- Fully Connected (FC) layer combines all the features learned in the preceding layers by connecting all the neurons, the last FC layer has the same number of outputs as the number of trained classes..

- The softmax layer is an activation function to normalize the FC layer output sum to one to utilize as a classification probability.

- Classification layer is the final layer to assign the softmax layer probabilities to each corresponding label.

2) Training option: The training data set should be split into batches data store containing data from different classes. DL CNN will train up through data batches in every epoch proceeding by shuffling the data to avoid over-fitting. Optionally, in the meanwhile, the classification accuracy could be validated via random input data.

\section{Bayesian Recursively enhancement}

In order to enhance the accuracy of the DL leak localization computed by means of Eq. (4) where only the current picture of pressure residuals $x^{k}$ is taken into account, previous classifications of pressure residual images can be considered applying recursively the Bayesian Theorem as was proposed in [9]. The Bayes Theorem

$$
P\left(l_{i} \mid x^{k}\right)=\frac{P\left(x^{k} \mid l_{i}\right) P\left(l_{i}\right)}{P\left(x^{k}\right)}, i=1, \ldots, n_{n}
$$


where $P\left(l_{i} \mid x^{k}\right)$ is the posterior probability, $P\left(l_{i}\right)$ is the prior probability and $P\left(x^{k}\right)$ is a normalization given by the total probability law

$$
P\left(x^{k}\right)=\sum_{i=1}^{n_{n}} P\left(x^{k} \mid l_{i}\right) P\left(l_{i}\right)
$$

allows the introduction of previous classification results by means the prior probability term $P\left(l_{i}\right)$ that can be considered as the posterior probability of previous time instant $k-1$. i.e. consider $P\left(l_{i}\right)=P\left(l_{i} \mid x^{k-1}\right)$ in Eq. (5). If (5) is computed recursively with this assumption in a time horizon of $H$ samples we obtain

$$
\begin{array}{r}
P\left(l_{i} \mid x^{k-H+n}\right)= \\
\frac{P\left(x^{k-H+n} \mid l_{i}\right) P\left(l_{i} \mid P\left(x^{k-H+n-1}\right)\right.}{P\left(x^{k-H+n}\right)}, \\
i=1, \ldots, n_{n}, n=1, \ldots, H
\end{array}
$$

Then, the most probable leak localization $\hat{\imath}$ can be computed as

$$
\hat{\imath}=\max _{i \in\left\{1, \ldots, n_{n}\right\}} P\left(l_{i} \mid x^{k}\right)
$$

where $P\left(l_{i} \mid x^{k}\right)$ is computed recursively from Eq. (7), and considering equalt probabilities for the posterior probabilities at time instant $H=0$.

The advantage of using (8) instead of (4) is that the information of pictures $x^{k-H+1}, \ldots, x^{k}$ is considered at time instant $k$ and some possible instantaneous classification errors due to uncertainties can be filtered.

\section{CASE STUDY}

The proposed leak localization approach has been applied to a simplified model of the WDN of Hanoi (Vietnam's capital) that has been studied in several previous leak localization works ( [16], [17], [7], [9], [5], [10]). This simplified model consists of one reservoir that supplies the inlet flow, 34 pipes and 31 inner nodes and its network graph is depicted in Fig. 4. It has been considered that sensor measurements are available every ten minutes but leak localization defined in (8) is computed every hour.

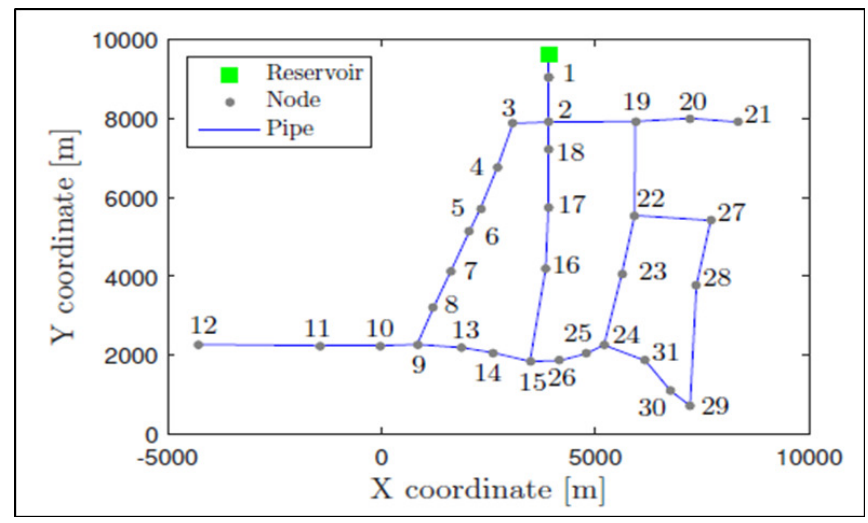

Fig. 4: Hanoi WDN.

\begin{tabular}{|c|c|c|}
\hline Configuration No. & $S_{N}$ & Installed sensors \\
\hline 1 & 4 & {$[2,20,25,29]$} \\
\hline 2 & 5 & {$[1,3,26,28,31]$} \\
\hline 3 & 6 & {$[1,3,12,26,28,29]$} \\
\hline 4 & 7 & {$[1,3,12,16,26,28,29]$} \\
\hline 5 & 8 & {$[1,3,12,15,16,26,28,29]$} \\
\hline 6 & 10 & {$[1,3,12,16,20,21,26,28,29,31]$} \\
\hline 7 & 12 & {$[1,3,6,12,16,20,21,25,26,28,29,31]$} \\
\hline
\end{tabular}

TABLE I: Sensor selection collaboration types

Seven different sensor configurations that consider a range from 4 to 12 pressure sensors installed in inner nodes of the network have been taken into account. The list of the seven sensor configurations and installed sensors is detailed in Table I.

It has been assumed that the inlet flow is also measured every 10 minutes and different daily patterns that range from 1100 to $4800[\mathrm{l} / \mathrm{s}]$ have been considered. The demand pattern in all demand nodes has been considered known but with an uncertainty of $\pm 5 \%$ of the estimated nodal demand value. Additive sensor noises in pressure measurements of $\pm 5 \%$ have been also considered. Finally, a leak with unknown magnitude but within 25 and $75[\mathrm{l} / \mathrm{s}]$, i.e. between $0.52 \%$ and $6.81 \%$ of total flow demand, has been considered.

In the next subsections the data generation process, the CNN training process and the leak localization performance assessment will be detailed.

\section{A. Data generation}

Data has been generated following the data generation scheme proposed in Section II-B. EPANET software [18] has been chosen to implement the hydraulic simulator of the network. Inlet flow patterns have been introduced to the simulator as well as actual and estimated user demands considering demand uncertainties described in the previous section. In particular, 30 daily flow patterns with a sample time of 10 minutes have been generated: 20 days for training data and 10 for testing data. The profile of the 30 daily flow patterns is shown in Fig. 5.

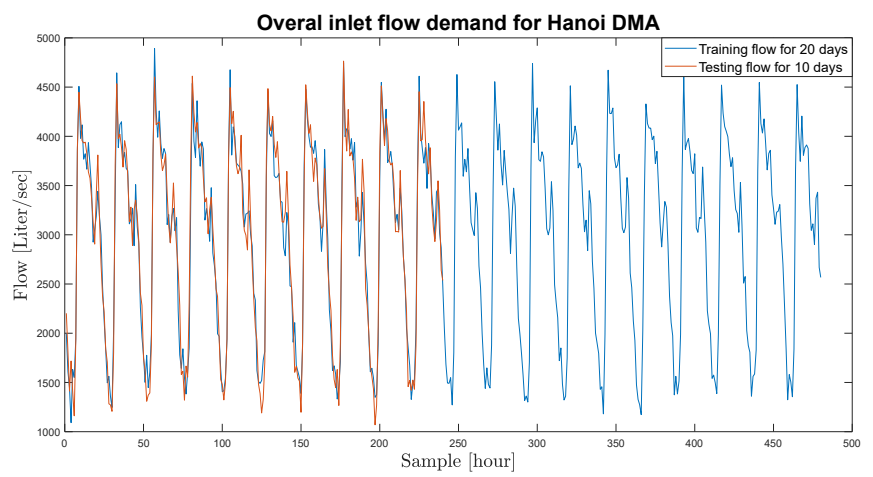

Fig. 5: Training and testing flow patterns.

According to data generation method presented in Fig. 2, additive noise of $\pm 5 \%$ has been added to pressure values computed by the hydraulic simulator that emulates the real WDN in the leak localization scheme depicted in Fig. 1. 
On the other hand, the 31 possible leak scenarios have considered in this simulator for the 30 daily flow patterns.

As leak localization will be computed every hour and pressure values are computed every ten minutes, the average value of residuals obtained in data generation method presented in Fig. 2 are computed and provided to the Kriging and picture generation process. In the Kriging and picture generation process, the seven different sensor configurations considered in the case study have been taken into account to generate seven different picture sets of training and validation data. The selected image resolution of generated images has been $100 \times 100$ i.e. $N_{I}=N_{J}=100$ in (2) for all sensor configurations.

As an example of the data obtained in the Kriging interpolation process, Fig. 6 depicts residual surface for leak 16 , and training flow corresponded to hour $10 A . M$. in day 4 computed by the Kriging interpolation considering sensor configuration 7 .

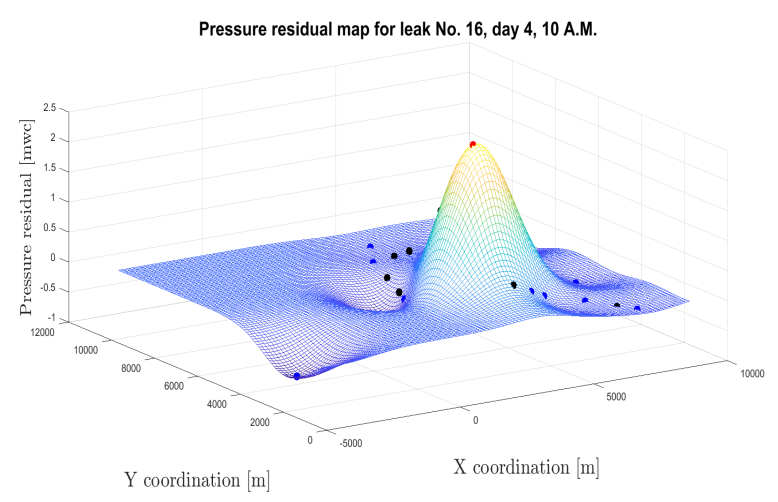

Fig. 6: A sample of pressure residual map.

On the other hand, as an example of the pictures obtained in the picture generation process several pictures are included in Table II computed for different inlet flow scenarios and for different leak scenarios.

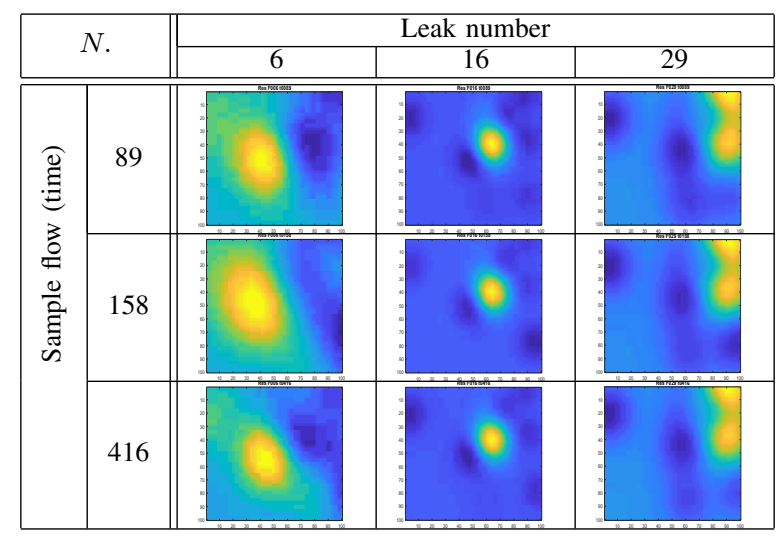

TABLE II: Residual Pressure pictures

As it can be noticed from the figures of Table II, there are several remarkable feature that are unique for the pictures that belong to a same leak scenario.

\section{B. Training process}

In this first attempt of applying DL for leak localization in WDNs the Deep Learning Toolbox ${ }^{\text {TM }}$ of MATLAB ${ }^{\circledR}$ has been used to train the CNN that performs the classification (leak localization). The prepared image data store containing 20 days of samples for each class (leak scenario) is used to train the designed structure of a deep CNN with suitable training settings. During the training process the $\mathrm{CNN}$ operation is validated using $15 \%$ of random data. The selected network architecture is composed of 21 layers that consists in 5 "Convolution" layers, 5 "batchNormalizationLayer" layers, 5 "ReLu" layers, 2 "maxpooling" layers, 1 "ImageInput" layer, 1 "fullyConnected" layer, 1 "SoftMax" layer and 1 "Classification" layer. The process of training has been repeated for the seven sensor configurations considered in the case study. Fig. 7 illustrates the training progress considering the data from sensor configuration 7 (12 sensors) detailed in Table I.

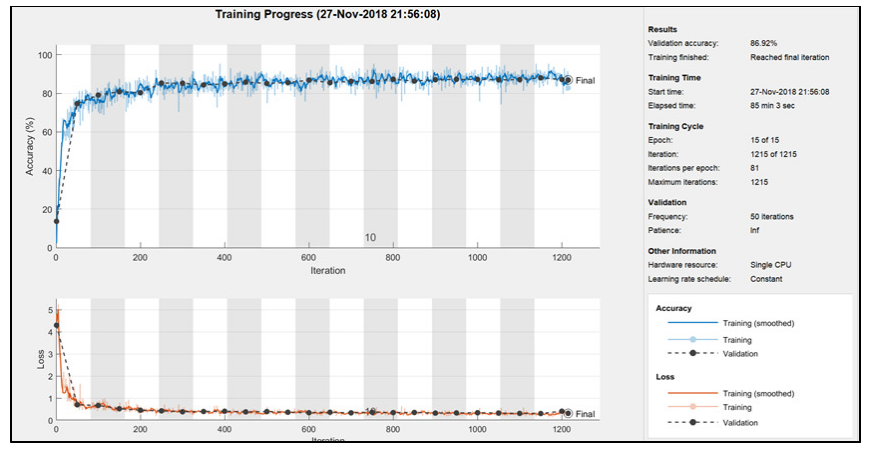

Fig. 7: CNN training process for the sensor configuration 7 (12 sensors) using the Deep Learning Toolbox ${ }^{\mathrm{TM}}$ of MATLAB $^{\circledR}$.

\section{Testing results}

Once the CNN model is calibrated, it can be evaluated by means of applying the pictures of the training set to the CNN classifier. In order to improve the performance in the leak localization task, the leak localization task has been computed by means of (8) with different time horizons $H=$ $1, \ldots, 24$.

To assess the performance of the leak localization approach, the confusion matrix $\Gamma\left(n_{n} \times n_{n}\right.$ matrix $)$ is used. The rows of this matrix correspond to the leak scenario and the columns to which leak is located $(\hat{l})$ by the leak localization method.

Given a particular confusion matrix $\Gamma$, the overall accuracy is defined as

$$
\mathrm{Ac}=\frac{\sum_{i=1}^{n_{n}} \Gamma_{i, i}}{\sum_{i=1}^{n_{n}} \sum_{j=1}^{n_{n}} \Gamma_{i, j}}
$$

The Average Topological Distance (ATD) proposed in [7] as a suitable indicator used to assess the leak localization performance in WDNs. The ATD is the average value of the minimum distance in nodes between the node with the leak 


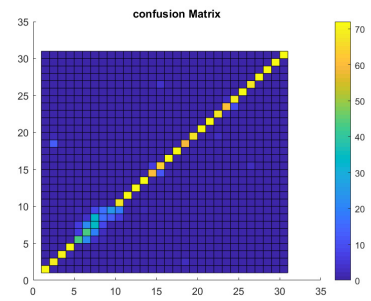

(a) $H=1$.

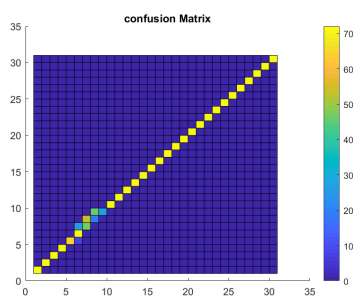

(b) $H=24$.
Fig. 8: Confusion matrix for sensor configuration 7 (12 sensors).

and the node candidate proposed by the leak localization method. The ATD is computed as follows

$$
\mathrm{ATD}=\frac{\sum_{i=1}^{n_{n}} \sum_{j=1}^{n_{n}} \Gamma_{i, j} \mathrm{~A}_{i, j}}{\sum_{i=1}^{n_{n}} \sum_{j=1}^{n_{n}} \Gamma_{i, j}}
$$

where $\mathbf{A}$ is a symmetric square matrix with size $n_{n}$ such that each element $\mathrm{A}_{i, j}$ contains the minimum topological distance in nodes between the nodes referred by indices $i$ and $j$.

The Accuracy and the ATD indicators obtained with the leak localization method considering $H=1$ and $H=24$ (one day of data) is summarized in Table for the seven different sensor configurations.

\begin{tabular}{|c|c|c|c|c|c|}
\hline & \multicolumn{2}{|c|}{ ACC. } & \multicolumn{2}{|c|}{ ATD } \\
\hline & & $\mathrm{H}=1$ & $\mathrm{H}=24$ & $\mathrm{H}=1$ & $\mathrm{H}=24$ \\
\hline \multirow{3}{*}{$\frac{0}{0}$} & 4 & 47.27 & 56.14 & 1.487 & 0.9969 \\
\hline & 5 & 56.09 & 66.26 & 0.8625 & 0.5269 \\
\hline & 6 & 74.33 & 81.41 & 0.3297 & 0.1994 \\
\hline \multirow{4}{*}{ 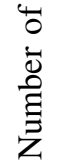 } & 7 & 75.59 & 81.05 & 0.2823 & 0.1465 \\
\hline & 8 & 77.91 & 89.2 & 0.2751 & 0.1080 \\
\hline & 10 & 82.21 & 91.58 & 0.2168 & 0.0842 \\
\hline & 12 & 87.81 & 94.13 & 0.1438 & 0.0586 \\
\hline
\end{tabular}

In addition, the confusion matrices obtained for the sensor configuration 7 (12 sensors) considering $H=1$ and $H=24$ are depicted in Fig. 8.

As it can be noticed from the results, as the number of sensors and the time horizon $H$ increase the performance indicators improve (AC increases and ATD decreases).

\section{CONCLUSION}

In this paper, a leak localization methodology in WDN based on Deep Learning CNN has been presented. Pressure residuals in some points of the network and interpolation using topological information of the network are used to generate pictures that can be evaluated by DL CNN image classification. Training and validation data is obtained in a off-line stage using a hydraulic simulators of the WDN where realistic errors as demand uncertainties and sensor noises are introduced. In this first attempt, the performance of the proposed method has been tested in to a simplified model of the WDN of Hanoi (Vietnam's capital). The Deep Learning Toolbox ${ }^{\mathrm{TM}}$ of MATLAB ${ }^{\circledR}$ has been used to train the CNN and the results obtained have been promising. These results encourage us to plan the improvement of the proposed method to deal with the problem of leak localization in real WDNs where the high number of nodes and uncertainties make a challenging problem. For this purpose more powerful Deep learning tools [19] will be necessary and picture generation and CNN architecture should be improved.

\section{REFERENCES}

[1] R. Puust, Z. Kapelan, D. A. Savić, T. Koppel, A review of methods for leakage management in pipe networks, Urban Water Journal 7 (1) (2010) 25-45.

[2] T. K. Chan, C. S. Chin, X. Zhong, Review of current technologies and proposed intelligent methodologies for water distributed network leakage detection, IEEE Access 6 (2018) 78846-78867. doi:10.1109/ACCESS.2018.2885444.

[3] R. Pérez, V. Puig, J. Pascual, J. Quevedo, E. Landeros, A. Peralta, Methodology for leakage isolation using pressure sensitivity analysis in water distribution networks, Control Engineering Practice 19 (10) (2011) 1157-1167.

[4] P. Cugueró-Escofet, J. Blesa, R. Pérez, M. A. Cugueró-Escofet, G. Sanz, Assessment of a leak localization algorithm in water networks under demand uncertainty, IFAC-PapersOnLine 48 (21) (2015) 226 231.

[5] J. Blesa, R. Pérez, Modelling uncertainty for leak localization in water networks, IFAC-PapersOnLine 51 (24) (2018) 730 - 735

[6] J. Mashford, D. de Silva, D. Marney, S. Burn, An approach to leak detection in pipe networks using analysis of monitored pressure values by support vector machine, in: Third International Conference on Network and System Security, 2009, pp. 534-539.

[7] A. Soldevila, J. Blesa, S. Tornil-Sin, E. Duviella, R. M. FernandezCanti, V. Puig, Leak localization in water distribution networks using a mixed model-based/data-driven approach, Control Engineering Practice 55 (2016) $162-173$.

[8] D. Wachla, P. Przystalka, W. Moczulski, A method of leakage location in water distribution networks using artificial neuro-fuzzy system, IFAC-PapersOnLine 48 (21) (2015) 1216 - 1223.

[9] A. Soldevila, R. M. Fernandez-Canti, J. Blesa, S. Tornil-Sin, V. Puig, Leak localization in water distribution networks using Bayesian classifiers, Journal of Process Control 55 (2017) 1-9.

[10] M. Quiones-Grueiro, J. M. B. de Lzaro, C. Verde, A. Prieto-Moreno, O. Llanes-Santiago, Comparison of classifiers for leak location in water distribution networks, IFAC-PapersOnLine 51 (24) (2018) 407 $-413$.

[11] L. Duan, M. Xie, J. Wang, T. Bai, Deep learning enabled intelligent fault diagnosis: Overview and applications, Journal of Intelligent and Fuzzy Systems 35 (5) (2018) 5771-5784.

[12] J. P. Kleijnen, Regression and kriging metamodels with their experimental designs in simulation: A review, European Journal of Operational Research 256 (1) (2017) 1 - 16.

[13] A. Soldevila, T. N. Jensen, J. Blesa, S. Tornil-Sin, R. M. FemandezCanti, V. Puig, Leak localization in water distribution networks using a kriging data-based approach, in: 2018 IEEE Conference on Control Technology and Applications (CCTA), 2018, pp. 577-582.

[14] J. Schmidhuber, Deep learning in neural networks: An overview, Neural Networks 61 (2015) 85 - 117.

[15] S. K. Zhou, H. Greenspan, D. Shen, Deep learning for medical image analysis, Academic Press, 2017.

[16] M. V. Casillas, V. Puig, L. E. Garza-Castañón, A. Rosich, Optimal Sensor Placement for Leak Location in Water Distribution Networks Using Genetic Algorithms, Sensors 13 (11) (2013) 14984-15005.

[17] L. Ferrandez-Gamot, P. Busson, J. Blesa, S. Tornil-Sin, V. Puig, E. Duviella, A. Soldevila, Leak localization in water distribution networks using pressure residuals and classifiers, IFAC-PapersOnLine 48 (21) (2015) $220-225$.

[18] L. A. Rossman, Epanet 2 user's manual, United States Envionmental Protection Agency.

[19] F. Chollet, Deep learning with python, Manning Publications Co., 2017. 